\title{
Pidotimod exacerbates allergic pulmonary infection in an OVA mouse model of asthma
}

\author{
LUO-QIN FU ${ }^{1,2^{*}}$, YA-LI LI ${ }^{1 *}$, AI-KUN FU ${ }^{1}$, YAN-PING WU ${ }^{1}$, \\ YUAN-YUAN WANG ${ }^{1}$, SHENG-LAN HU ${ }^{3}$ and WEI-FEN LI ${ }^{1}$
}

\begin{abstract}
${ }^{1}$ Key Laboratory of Molecular Animal Nutrition and Feed Sciences, College of Animal Science, Zhejiang University, Hangzhou, Zhejiang 310058; ${ }^{2}$ Clinical Research Institute, Zhejiang Provincial People's Hospital, Hangzhou, Zhejiang 310014;

${ }^{3}$ Institute of Animal Science, Guangdong Academy of Agricultural Sciences, Guangzhou, Guangdong 510640, P.R. China
\end{abstract}

Received September 3, 2016; Accepted May 16, 2017

DOI: $10.3892 / \mathrm{mmr} .2017 .7046$

\begin{abstract}
Pidotimod is a synthetic dipeptide with biological and immuno-modulatory properties. It has been widely used for treatment and prevention of recurrent respiratory infections. However, its impact on the regulation of allergic pulmonary inflammation is still not clear. In the current study, an ovalbumin (OVA)-induced allergic asthma model was used to investigate the immune-modulating effects of pidotimod on airway eosinophilia, mucus metaplasia and inflammatory factor expression compared with dexamethasone (positive control). The authors determined that treatment with pidotimod exacerbated pulmonary inflammation as demonstrated by significantly increased eosinophil infiltration, dramatically elevated immunoglobulin E production, and enhanced T helper 2 response. Moreover, treatment failed to attenuate mucus production in lung tissue, and did not reduce OVA-induced high levels of FIZZ1 and Arg1 expression in asthmatic mice. In contrast, administration of dexamethasone was efficient in alleviating allergic airway inflammation in OVA-induced asthmatic mice. These data indicated that pidotimod as an immunotherapeutic agent should be used cautiously and the
\end{abstract}

Correspondence to: Professor Wei-Fen Li, Key Laboratory of Molecular Animal Nutrition and Feed Sciences, College of Animal Science, Zhejiang University, 866 Yu Hangtang Road, Hangzhou, Zhejiang 310058, P.R. China

E-mail: weifenli@zju.edu.cn

Dr Sheng-Lan $\mathrm{Hu}$, Institute of Animal Science, Guangdong Academy of Agricultural Sciences, 1 Dafeng 1st Street, Wushan, Tianhe, Guangzhou, Guangdong 510640, P.R. China

E-mail: hsl_0517@163.com

${ }^{*}$ Contributed equally

Abbreviations: HE stain, hematoxylin and eosin; OVA, ovalbumin; $\mathrm{NO}$, antimicrobial nitric oxide; BAL, bronchoalveolar lavage; PAS stain, periodic acid-Schiff stain

Key words: pidotimod, asthma, eosinophils, mucus, FIZZ1, Arg1, inflammation effectiveness for controlling allergic asthma needs further evaluation and research.

\section{Introduction}

Allergic asthma is a chronic inflammatory disease characterized by reversible airflow obstruction, bronchial hyperreactivity, elevated serum immunoglobulin E (IgE) levels and airway eosinophilia (1). T-helper 1 (Th1)/Th2 cytokine imbalance is critical for the initiation and perpetuation of allergic asthma $(2,3)$. Th2 cytokine interleukins (ILs) IL-4, IL-5 and IL-13 skew lung macrophage differentiation to M2 type (alternatively activated macrophage). M2 macrophages secrete proteins that enhance asthma pathology upon allergen challenge in asthmatic mice. These proteins include found in inflammatory zone-1 (FIZZ1), chitinase-like protein 3 (Ym-1) and arginase $1(\operatorname{Arg} 1)(4)$.

Treatments and interventions for asthma have improved substantially in recent years. Currently, glucocorticoid inhalation is the most effective therapy available (5). Systemic administration of glucocorticoids, such as dexamethasone, attenuates eosinophilia in target tissues (6) and downregulates chemokine expression (7), thereby preventing asthma exacerbation. While current therapies are effective in temporarily relieving symptoms by suppressing inflammation, they are ineffective in treating the chronic course of disease (8). Furthermore, prolonged use of inhaled corticosteroids may have adverse side effects (9). Thus, more effective treatments and therapeutic strategies are needed.

Recently, pidotimod (3-L-pyroglutamyl-L-thiaziolidine4-carboxylic acid) has been considered as a potential therapy for allergic asthma. Pidotimod is a synthetic dipeptide prepared from L-cysteine and L-pyroglutamic acid with biological and immunological activity on innate and adaptive immune responses (10). Up to now, clinical studies on this dipeptide have focused on its use as a treatment for respiratory tract infections in children $(11,12)$. Previous studies have demonstrated that pidotimod modulates airway epithelial cell functions (13), enhances natural killer cell activity (14) and induces dendritic cell maturation (15). However, the impact of pidotimod on the regulation of allergic pulmonary inflammation is still not clear. Recently, the authors demonstrated that pidotimod is sufficient 
to facilitate IL-4-induced M2 macrophage polarization (16), suggesting that pidotimod may enhance cytokine activity and exacerbate pulmonary inflammation. In the current study, the authors investigated the effects of pidotimod on allergic lung inflammation. An ovalbumin (OVA)-induced allergic asthma model was used to investigate the immune-modulating effects of pidotimod on airway eosinophilia, mucus metaplasia and allergic inflammation in comparison to dexamethasone (positive control).

\section{Materials and methods}

Animals. Twenty-eight female C57BL/6 mice (6- to 7-week-old) were purchased from the Shanghai SLAC Laboratory Animal Co., Ltd. (Shanghai, China). Animals were maintained in a specific pathogen-free facility and provided with food and water ad libitum. All animal experiments were approved by the Institutional Animal Care and Use Committee of Zhejiang University (Hangzhou, China).

Induction of allergic asthma and drug treatments. Allergic asthma was induced by a modified protocol described by Shen et al (17). Mice were sensitized by subcutaneous injection on day 0 with $40 \mu \mathrm{g}$ OVA (Grade V, Sigma-Aldrich; Merck KGaA, Darmstadt, Germany) in $0.1 \mathrm{ml}$ Imject Alum Adjuvant (Thermo Fisher Scientific, Inc., Waltham, MA, USA), followed by a booster injection with the same amount of OVA and alum on day 14. At 10 days following the last injection, sensitized mice were subsequently challenged with $1 \%$ OVA in saline by nebulization for $40 \mathrm{~min}$ daily for three days (OVA group). Mice were treated by an intraperitoneal injection of $0.5 \mathrm{mg} / \mathrm{kg}$ dexamethasone (Boston Biomedical, Inc., Cambridge, MA, USA; OVA + Dex group) or by oral gavage with $200 \mathrm{mg} / \mathrm{kg}$ pidotimod (Santa Cruz Biotechnology, Inc., Dallas, TX, USA) (OVA + Pido group) $1 \mathrm{~h}$ prior to OVA inhalation challenge. Control mice were given saline in place of OVA in both the sensitization and the challenge stages. All mice were sacrificed $24 \mathrm{~h}$ following the last challenge.

Bronchoalveolar lavage and cellular differentials. Bronchoalveolar lavage (BAL) was obtained by instilling and withdrawing $2 \mathrm{ml}$ saline via a tracheal cannula. Cells in the BAL were washed and counted before centrifugation (at 800 $\mathrm{x} \mathrm{g}$ ) onto microscope slides using cytospin techniques. After staining with Wright-Giemsa (Polysciences, Inc., Warrington, PA, USA), relative numbers of lymphocytes and eosinophils were determined based on the morphological criteria. A total of 300 cells were counted under light microscopy.

Serum IgE and BAL cytokine analysis. Blood samples were obtained from tail veins. Total serum IgE (catalog no. 88-50460; eBioscience, Thermo Fisher Scientific, Inc.) and cytokine including IL-4 (catalog no. 88-7044; eBioscience, Thermo Fisher Scientific, Inc.), IL-5 (catalog no. 88-7054; eBioscience, Thermo Fisher Scientific, Inc.) and IL-13 (catalog no. 88-7137; eBioscience, Thermo Fisher Scientific, Inc.) levels in BAL fluid were measured by ELISA according to manufacturer's protocol. The levels of IgE, IL-4, IL-5 and IL-13 were expressed as $\mathrm{pg} / \mathrm{ml}$ using a standard curve.
Histopathology and immunohistochemistry. Following BAL sampling, lung tissues were fixed in $10 \%$ neutral buffered formalin, paraffin-embedded, sectioned into $4 \mu \mathrm{m}$ slices and stained with haematoxylin and eosin for examination of inflammatory cell infiltration. The degree of perivascular and peribronchial inflammation was graded as none to severe on a 0 to 4 scale, as described previously (18). Periodic acid-Schiff (PAS)-stained goblet cells in airway epithelium were measured double blind using a numerical scoring system (19). Individual airway scores were summed from each mouse to generate an overall histopathology score. For FIZZ1 and Arg1 expression was examined by incubating de-paraffinized sections with rabbit anti-mouse FIZZ1 primary antibody (catalog no. ab39626; Abcam, Cambridge, MA, UK; 1:100 dilution) or anti-Arg1 primary antibody (catalog no. AV45673; Sigma-Aldrich, Merck KGaA; 1:200 dilution) overnight at $4^{\circ} \mathrm{C}$. Incubation was followed by a second incubation with polyclonal goat anti-rabbit immunoglobulins/horseradish peroxidase (catalog no. A9169; Sigma-Aldrich; Merck KGaA; 1:400 dilution) for $2 \mathrm{~h}$ at room temperature. The nuclei were counterstained with hematoxylin.

Statistical analysis. All results are expressed as the mean \pm standard deviation. Statistical significance was determined using two-tailed Student's t-test with GraphPad Prism (GraphPad Software, Inc., La Jolla, CA, USA). P<0.05 was considered to indicate a statistically significant difference.

\section{Results}

Pidotimod increased OVA-induced eosinophil infiltration. To evaluate the impact of pidotimod on asthma, the authors first examined inflammatory cell infiltration in BAL fluid (BALF) samples from OVA-immunized mice. The results demonstrated that compared with control group, OVA sensitization and challenge induced a significant increase in total cell numbers (Fig. 1A) and eosinophil recruitment (Fig. 1B) into the BALF. As predicted, mice treated with dexamethasone (Dex + OVA group), an effective therapy for asthma, reported a profound decrease in eosinophils (Fig. 1B) and a slight decrease in total cell counts compared to OVA group (Fig. 1A). In contrast, pidotimod-treated mice (Pido + OVA group) had a significantly higher number of eosinophils in the BALF, compared to mice treated with OVA alone (Fig. 1B). A trend of increased total cell numbers was also observed in mice treated with pidotimod; however, there was no statistical significance (Fig. 1A).

Pidotimod enhances $I g E$ production and Th2 response in OVA model of asthma. The immunologic response to allergen challenge in allergic asthma is characterized by elevated IgE and Th2 -related cytokine secretion (20). Indeed, mice sensitized and challenged with OVA had strongly elevated levels of total serum IgE (Fig. 2A) and produced significant amounts of Th2-cytokines (IL-4, IL-5 and IL-13) when compared to controls. Administration of dexamethasone before OVA sensitization markedly reduced serum IgE, IL-4 and IL-5 levels in BALF compared with untreated OVA-sensitized mice. Interestingly, both serum IgE and BALF IL-5 synthesis was clearly enhanced following pidotimod application 

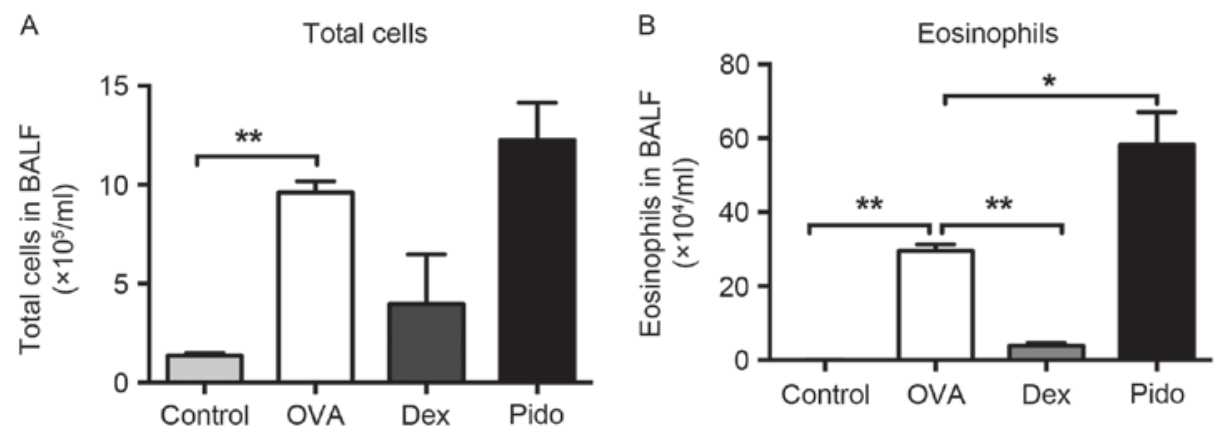

Figure 1. Cell differentiation in BALF of OVA-immunized mice. BALF samples were collected $24 \mathrm{~h}$ after the last OVA aerosol challenge. (A) Total cell numbers and (B) eosinophils in BALF were counted. Results are expressed as mean \pm standard deviation, $(\mathrm{n}=6)$ and are representative of three independent experiments. ${ }^{*} \mathrm{P}<0.05,{ }^{* *} \mathrm{P}<0.01,{ }^{* * * *} \mathrm{P}<0.001$ as indicated. BALF, bronchoalveolar lavage fluid; OVA, ovalbumin; Dex, dexamethasone; Pido, pidotimod.

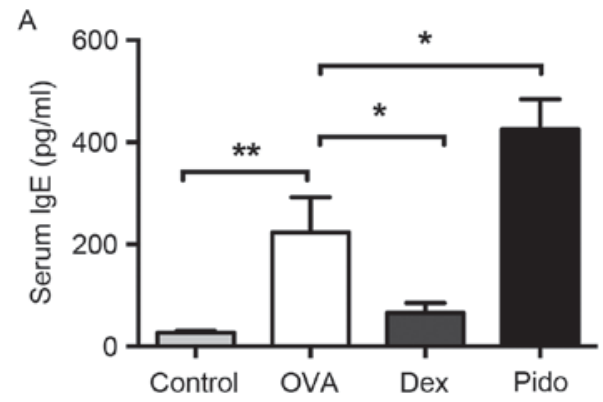

C

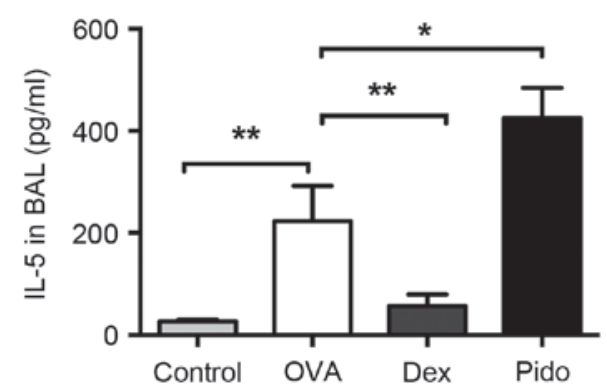

B

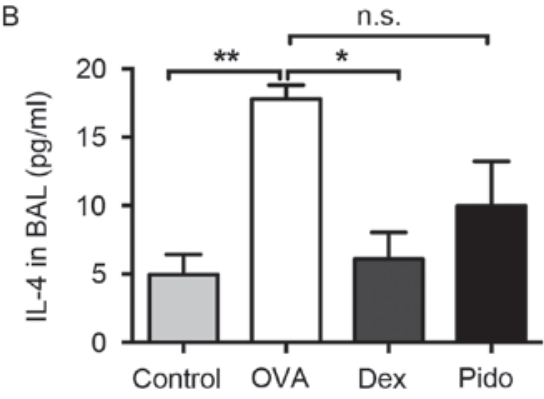

D

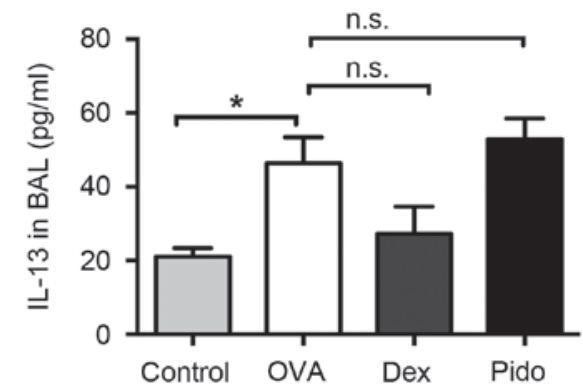

Figure 2. Total serum IgE and T helper 2 cytokines production in BALF of OVA-immunized mice. Blood and BALF samples were collected $24 \mathrm{~h}$ following the final OVA challenge to analyze the levels of total serum (A) IgE, (B) IL-4, (C) IL-5 and (D) IL-13 in BALF by ELISA. Data are presented as mean \pm standard deviation ( $\mathrm{n}=5$ or 6 in each group) ${ }^{*} \mathrm{P}<0.05,{ }^{* *} \mathrm{P}<0.01,{ }^{* * * *} \mathrm{P}<0.001$ as indicated. IgE, immunoglobulin E; BALF, bronchoalveolar lavage fluid; OVA, ovalbumin; IL, interleukin; Dex, dexamethasone; Pido, pidotimod.

(Fig. 2A and C). Levels of IL-4 and IL-13 were not modified by pidotimod treatment (Fig. 2B and D). These data suggested that pidotimod exacerbates the imbalanced pulmonary immune response in OVA-immunized mice.

Pidotimod fails to attenuate pulmonary histological changes and mucus metaplasia associated with OVA-induced asthma. Histological examination of the extent and anatomical distribution of allergic inflammation revealed intense inflammation in the lungs of OVA-treated mice. The bronchi and vessels were surrounded by inflammatory infiltrates and were frequently lined with a hyperplastic epithelium (Fig. 3B). Dexamethasone administration $1 \mathrm{~h}$ before OVA sensitization and challenge significantly decreased cellular infiltration of airways (Fig. 3C and E) when compared to infiltration in the airways of untreated OVA-immunized mice (Fig. 3B). However, there was no appreciable difference in the degree of tissue inflammation observed between the OVA group (Fig. 3B) and OVA + Pido group (Fig. 3D), with OVA-immunized mice and pidotimod-treated mice presenting similar histological scores (Fig. 3E).

In addition to increased inflammatory infiltrates, goblet cell metaplasia and mucus production are also indicative of the degree of inflammation. Lung PAS staining demonstrated an upregulation of mucus glycoprotein production and basement membrane thickening in OVA sensitized and challenged mice (Fig. 4G). Immunomodulation in dexamethasone-treated mice was evident by a marked reduction in the secretion of mucus (Fig. $3 \mathrm{H}$ and $\mathrm{J}$ ) compared to untreated OVA-sensitized mice (Fig. 3G). In contrast, mucus production was not significantly altered following treatment with pidotimod (Fig. 3I and J). No airway inflammation or mucus production was identified in control mice (Fig. 3A and F). Together, these data demonstrated that both 
HE staining
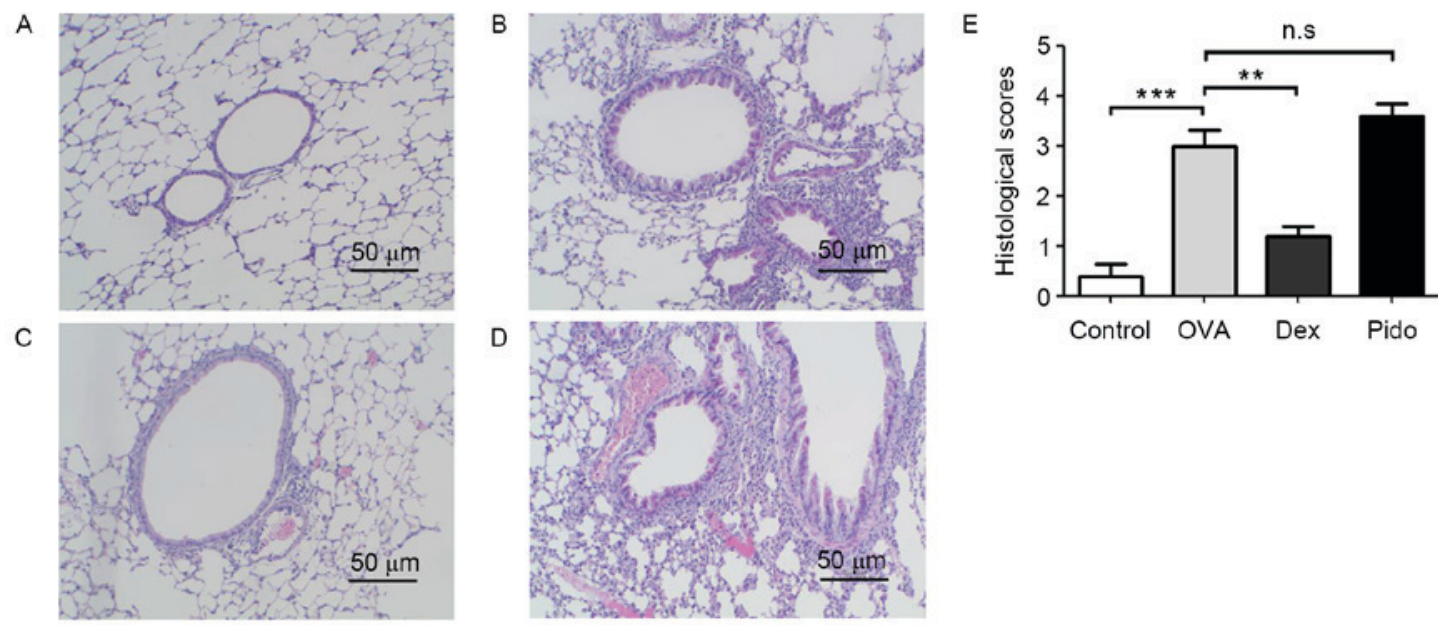

PAS staining

F

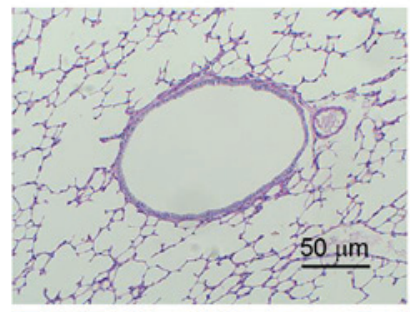

$\mathrm{H}$

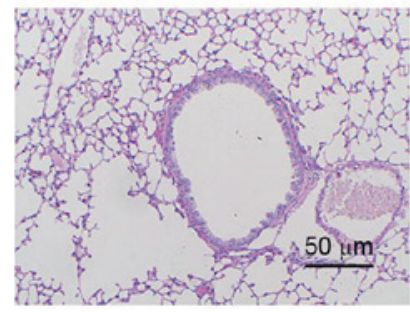

G

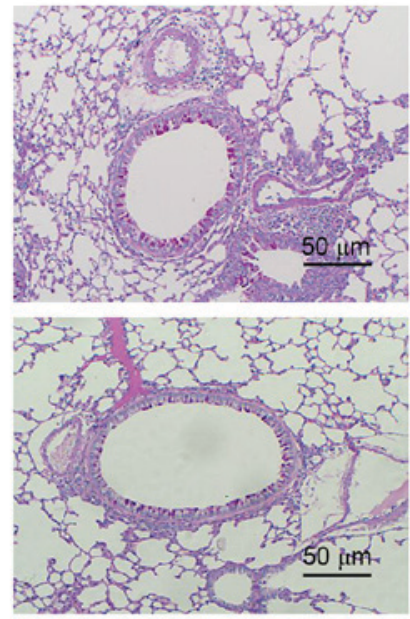

J

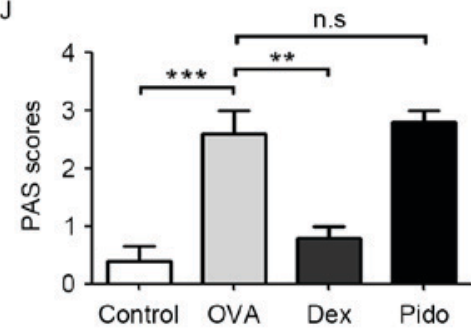

Figure 3. Pidotimod fails to attenuate pulmonary histological changes and mucus metaplasia associated with OVA treatment. An assessment of pulmonary inflammation was made by determining the extent of inflammatory infiltrates in sections stained with HE (A, magnification, x400) or PAS (B, magnification, x400). Representative photomicrographs of stained lung sections from (A and F) control group, (B and G) OVA-challenged group, (C and H) Dex-treated group (OVA + Dex) and (D and I) Pido-treated group (OVA + Pido) are shown. (E and J) Stained sections were scored as described in Materials and methods (mean \pm standard deviation; $\mathrm{n}=5-6 /$ group). ${ }^{*} \mathrm{P}<0.05,{ }^{* *} \mathrm{P}<0.01,{ }^{* * *} \mathrm{P}<0.001$ as indicated. OVA, ovalbumin; HE, hematoxylin eosin; PAS, periodic acid-Schiff; Dex, dexamethasone; Pido, pidotimod.

pulmonary histological changes and mucus metaplasia was not attenuated by pidotimod treatment.

Pidotimod is unable to down-regulate the OVA-induced FIZZ1 expression. FIZZ1 serves an important role in airway remodeling and development of pulmonary inflammation (21). To further study the potential influence of pidotimod on OVA-induced inflammation, the authors next analyzed FIZZ1 expression in OVA-induced asthmatic mice by immunohistochemistry. As shown in Fig. 4B and E, the expression levels of FIZZ1 in airway epithelium cells were significantly enhanced in OVA-immunized mice when compared to control mice (Fig. 4A). However, there was a marked reduction of FIZZ1 expression in dexamethasone-treated mice (Fig. 4C) when compared to untreated OVA-immunized mice (Fig. 4B). Notably, pidotimod-exposed mice maintained a high expression level of FIZZ1 (Fig. 4D), suggesting that pidotimod administration is unable to effectively reduce FIZZ1 expression in OVA-induced asthmatic mice.

Pidotimod is unable to downregulate the OVA-induced Arg1 expression. Arg1 has been suggested to serve a vital role in asthma development, severity and progression (22). The authors evaluated the impact of pidotimod on Arg1 expression in OVA-induced murine models of asthma. A clear enhancement of Arg1 expression in OVA-immunized mice was identified (Fig. 5B), whereas dexamethasone-treated mice exhibited a dramatic reduction in Arg1 expression (Fig. 5C) compared to untreated OVA mice. By contrast, the expression levels of Arg1 in the lungs of pidotimod-treated mice were comparable to the levels in OVA mice (Fig. 5D and E). These observations indicated that pidotimod fails to downregulate Arg1 expression in the OVA model of asthma. 
A

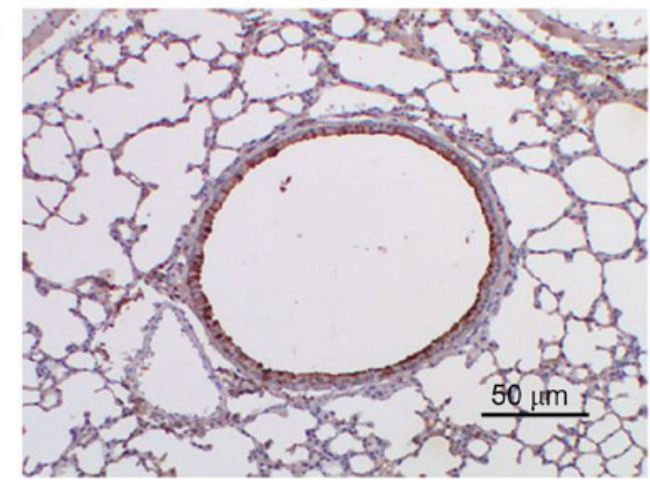

C

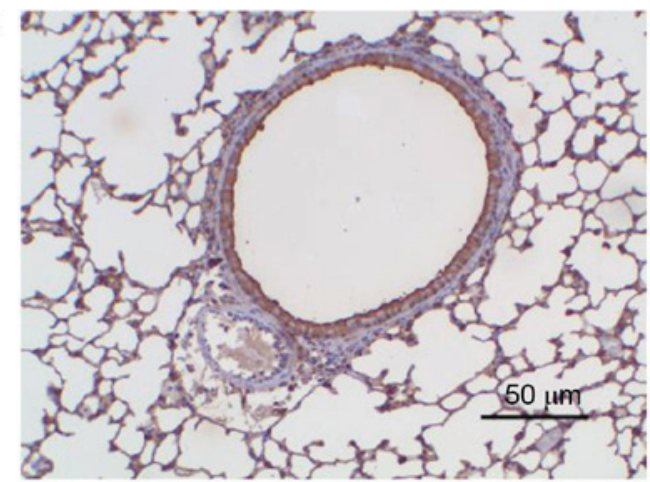

B

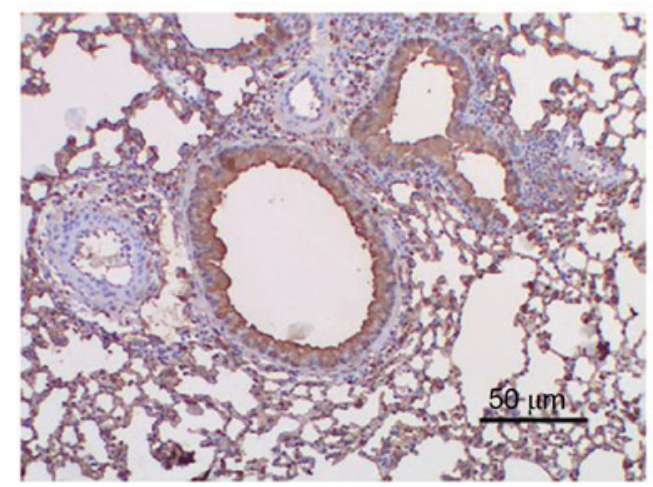

D

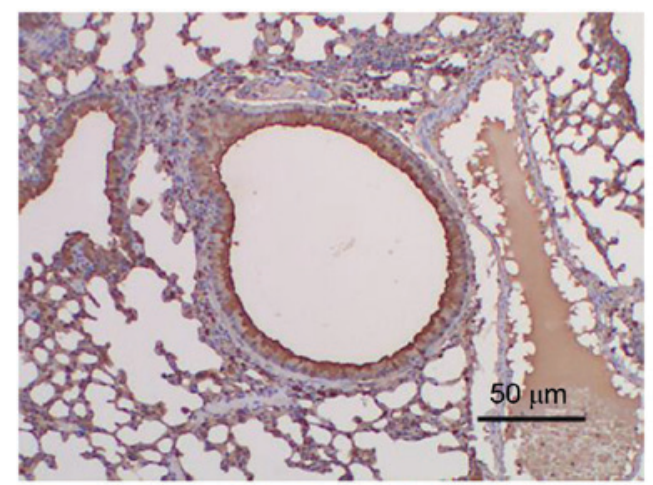

E

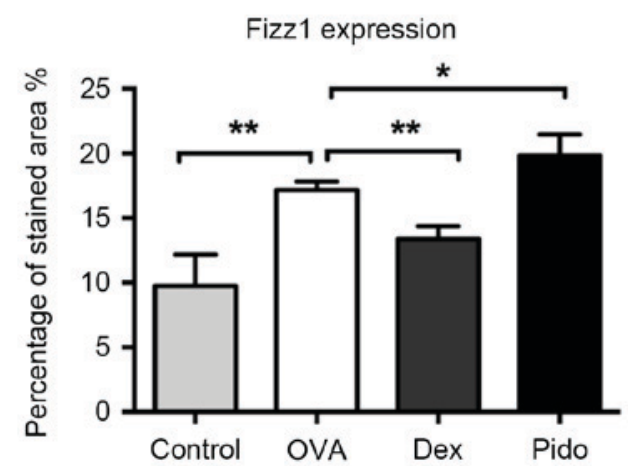

Figure 4. Immunohistochemistry analysis of FIZZ1 expression in lung tissues. Representative photographs from (A) control group, (B) OVA-challenged group, (C) Dex-treated group (OVA + Dex) and (D) Pido-treated group (OVA + Pido) are shown. All images are presented at x400 magnification. (E) Stained sections were scored (mean \pm standard deviation; $\mathrm{n}=5$-6/group). ${ }^{*} \mathrm{P}<0.05,{ }^{* *} \mathrm{P}<0.01,{ }^{* * * *} \mathrm{P}<0.001$ as indicated. FIZZ1, found in inflammatory zone-1; OVA, ovalbumin; Dex, dexamethasone; Pido, pidotimod.

\section{Discussion}

Prevalence of asthma and allergies has increased over the last few decades (23). Long-term use of inhaled corticosteroids, especially at high doses, may cause systemic or local side effects $(9,24)$. Pidotimod is a synthetic dipeptide with biological and immuno-modulatory properties that is largely used for treatment and prevention of recurrent respiratory infections (12), suggesting a potential therapeutic role for pidotimod in allergic pulmonary inflammation.

In 2013, the authors demonstrated that pidotimod is sufficient to facilitate IL-4-induced M2 macrophage polarization and improve macrophage functioning (13), suggesting that pidotimod may exacerbate inflammation. In the current study, the impact of pidotimod in the regulation of allergic inflammation was investigated using an OVA murine model of asthma, a widely used model of asthma due to resemblance to human asthma pathology and pathophysiology $(5,25)$.

As expected, exposure to OVA was sufficient to induce pulmonary allergic inflammation as characterized by increased eosinophil recruitment, elevated serum IgE production and enhanced Th2-cytokines (IL-4, IL-5 and IL-13) synthesis, which was similar in many respects to human allergic asthma. Systemic of administration glucocorticoids has been commonly used in treating patients with acute asthma $(5,26)$. Dexamethasone, a synthetic glucocorticoid, was efficient in alleviating allergic airway inflammation as manifested by reduced inflammatory infiltration, decreased serum OVA-specific IgE level, downregulated expression of acidic mammalian chitinase and gob-5, and inhibit chitinase bioactivity in mice with preexisting OVA generated experimental asthma $(27,28)$. Pidotimod treatment significantly 
A

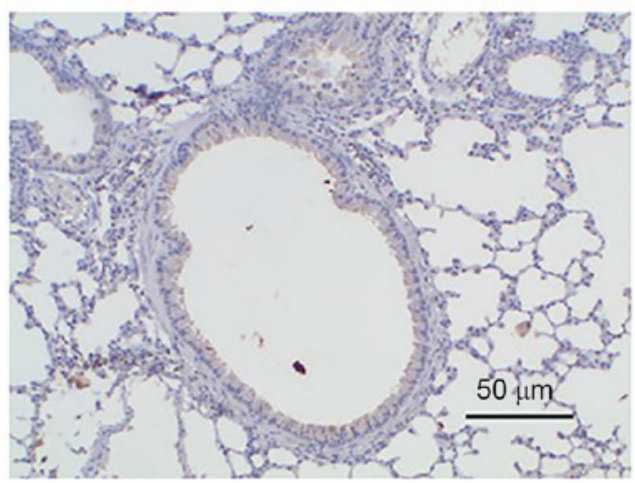

C

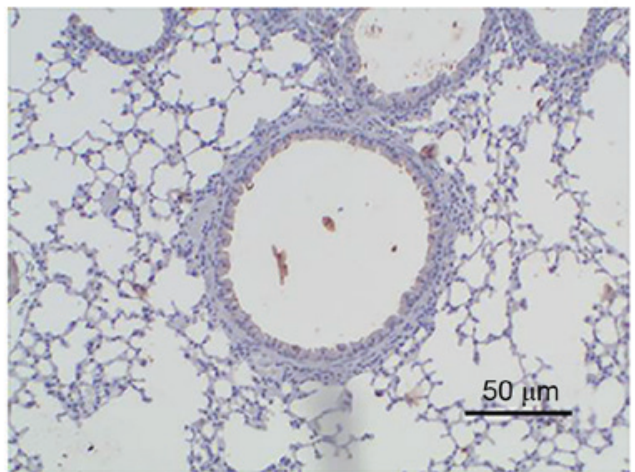

B

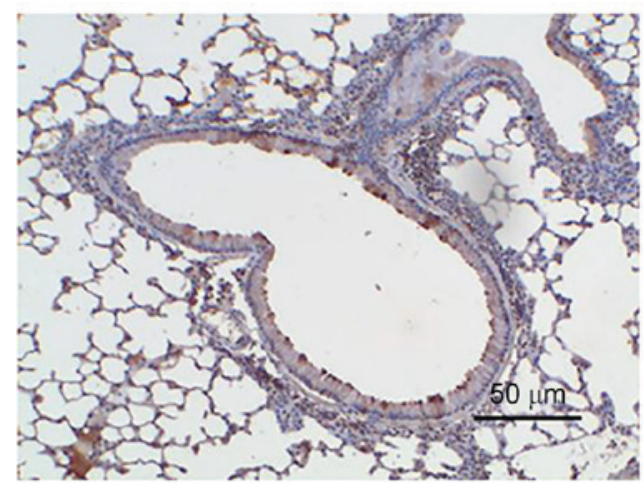

D

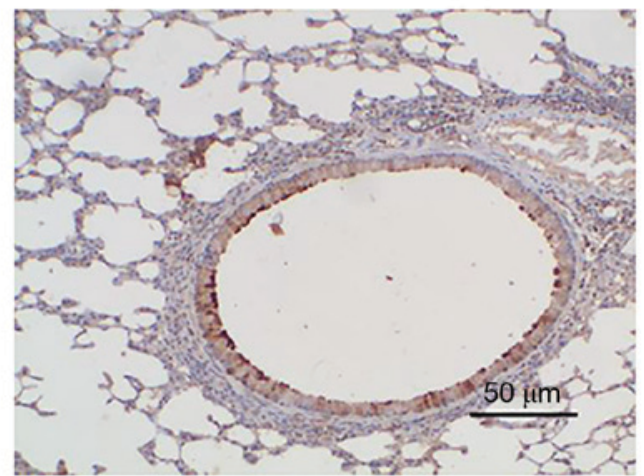

E

Arg1 expression

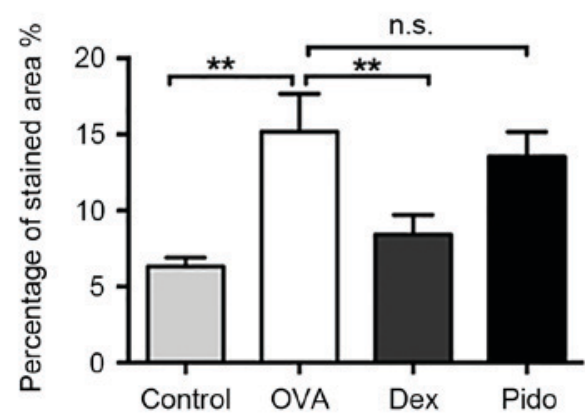

Figure 5. Immunohistochemistry analysis of Arg1 expression in lung tissues. Representative photographs from (A) control group, (B) OVA-challenged group, (C) Dex-treated group (OVA + Dex) and (D) Pido-treated group (OVA + Pido) are shown. All images are presented at x400 magnification. (E) Stained sections were scored (mean \pm standard deviation; $\mathrm{n}=5$-6/group). ${ }^{*} \mathrm{P}<0.05,{ }^{* *} \mathrm{P}<0.01,{ }^{* * * *} \mathrm{P}<0.001$ as indicated. OVA, ovalbumin; Dex, dexamethasone; Pido, pidotimod.

increased eosinophil infiltration in BALF and dramatically enhanced IgE production and Th2 response in OVA-induced asthmatic mice. This observation is in disagreement with a previous study reporting that the addition of pidotimod resulted in no differences in IL-4 production in peripheral blood mononuclear cells from asthmatic children (29).

Mucus gland hyperplasia depends on a Th2-biased airway inflammation (30). Th2 directly enhances Th2 cytokine-mediated goblet cell hyperplasia and increased mucus formation in the airways of asthmatic mice (31). In the present study, a profound increase in mucus glycoprotein production and basement membrane thickening was observed in OVA-immunized mice, which were reduced by dexamethasone treatment. However, mucus production was not significantly altered after treatment with pidotimod. Additionally, the combination of pidotimod and dexamethasone was indicated to significantly attenuate pathological changes and mucus production in asthmatic mice (data not shown). Together, these data suggested that pidotimod administration is unable to effectively suppress the development of allergic airway inflammation in OVA-induced asthmatic mice.

FIZZ1 was first reported in 2000 by Holcomb et al (32) in allergic pulmonary inflammation and was shown to promote airway remodeling (33). Previous studies revealed that FIZZ1 expression was markedly increased in hypertrophic, hyperplastic bronchial epithelium and in alveolar type II epithelial cells during allergic airway inflammation $(32,34)$. FIZZ1 also promotes pulmonary vascular smooth muscle cell proliferation (35).

In the current study, the expression level of FIZZ1 in airway epithelium cells was significantly enhanced in OVA-immunized mice compared with control mice. These results are in line with previous findings indicating that FIZZ1 is upregulated in lung tissues of asthmatic mice $(21,36)$. In addition, dexamethasone reduced FIZZ1 expression in OVA-immunized mice, the high level of FIZZ1 expression 
was maintained in pidotimod-exposed mice, suggesting that pidotimod administration has no influence on the regulation of FIZZ1 in OVA-induced asthmatic mice.

Arg1, which hydrolyses L-arginine into ornithine and urea, has been identified as a key player in the pathophysiology of asthma $(37,38)$. An enhanced level of arginase has been observed in airways and lung tissue obtained from animal models of allergic asthma (39) and from patients (40). It has been demonstrated that increased arginase activity may contribute to allergen-induced airway remodeling, inflammation and hyperresponsiveness in asthma (41). The expression of arginase was strongly induced by Th2 cytokines $(37,41,42)$. Associated with the enhanced Th2 response in OVA-immunized mice in this study, the Arg1 expression was significantly elevated in the lung, which was consistent with previous findings $(41,43)$. While treatment with dexamethasone decreased the expression of Arg1 and alleviated allergic airway inflammation in OVA-induced asthmatic mice, Arg1 expression was unaffected by pidotimod treatment. These results are in line with a recent study, demonstrating that pidotimod increase M2 marker gene expression (FIZZ1 and Arg1) in Th2 cytokine induced M2 macrophage, thereby facilitating macrophage polarization (16).

In conclusion, the current findings indicated that pidotimod treatment exacerbates pulmonary inflammation as demonstrated by increased eosinophil infiltration, elevated IgE production and enhanced Th2 response, while it fails to attenuate mucus production in lung tissue. Moreover, the high levels of FIZZ1 and Arg1 expression were maintained in asthmatic mice with pidotimod administration. Thus, the results indicated that pidotimod as an immunotherapeutic agent should be used cautiously and the effectiveness for controlling allergic asthma needs further evaluation and research.

\section{Acknowledgements}

The present work was supported by the grants from the planning subject of 'the Twelfth Five-year-plan' in National Science and Technology for the Rural Development in China (grant no. 2013BAD10B03) and the National Natural Science Foundation of China (grant no. 81570198).

\section{References}

1. Wenzel SE: Asthma: Defining of the persistent adult phenotypes. Lancet 368: 804-813, 2006.

2. Barnes PJ, Chung KF and Page CP: Inflammatory mediators of asthma: An update. Pharmacol Rev 50: 515-596, 1999.

3. Holt PG, Macaubas C, Stumbles PA and Sly PD: The role of allergy in the development of asthma. Nature 402 (6760 Suppl): B12-B17, 1999.

4. Balhara J and Gounni AS: The alveolar macrophages in asthma: A double-edged sword. Mucosal Immunol 5: 605-609, 2012.

5. Ni Z, Tang J, Cai Z, Yang W, Zhang L, Chen Q, Zhang L and Wang X: A new pathway of glucocorticoid action for asthma treatment through the regulation of PTEN expression. Respir Res 12: 47, 2011

6. Baatjes AJ, Sehmi R, Saito H, Cyr MM, Dorman SC, Inman MD, O'Byrne PM and Denburg JA: Anti-allergic therapies: Effects on eosinophil progenitors. Pharmacol Ther 95: 63-72, 2002.

7. Jahnsen FL, Haye R, Gran E, Brandtzaeg P and Johansen FE: Glucocorticosteroids inhibit mRNA expression for eotaxin, eotaxin-2, and monocyte-chemotactic protein-4 in human airway inflammation with eosinophilia. J Immunol 163 1545-1551, 1999.
8. Akdis CA: Therapies for allergic inflammation: Refining strategies to induce tolerance. Nat Med 18: 736-749, 2012.

9. Dahl R: Systemic side effects of inhaled corticosteroids in patients with asthma. Respir Med 100: 1307-1317, 2006.

10. Riboldi P, Gerosa M and Meroni PL: Pidotimod: A reappraisal. Int J Immunopathol Pharmacol 22: 255-262, 2009.

11. Licari A, De Amici M, Nigrisoli S, Marseglia A, Caimmi S, Artusio L and Marseglia GL: Pidotimod may prevent recurrent respiratory infections in children. Minerva Pediatr 66: 363-367, 2014.

12. Namazova-Baranova LS, Alekseeva AA, Kharit SM, Kozhevnikova TN, Taranushenko TE, Tuzankina IA and Scarci F: Efficacy and safety of pidotimod in the prevention of recurrent respiratory infections in children: A multicentre study. Int J Immunopathol Pharmacol 27: 413-419, 1900.

13. Carta S, Silvestri M and Rossi GA: Modulation of airway epithelial cell functions by Pidotimod: NF-kB cytoplasmatic expression and its nuclear translocation are associated with an increased TLR-2 expression. Ital J Pediatr 39: 29, 2013.

14. Migliorati G, Nicoletti I and Riccardi C: Immunomodulating activity of pidotimod. Arzneimittelforschung 44: 1421-1424, 1994.

15. Hu X, Zhang W, Wang L, Wan N, Wang B, Li W, Hua H, Hu X and Shan F: The detailed analysis of the changes of murine dendritic cells (DCs) induced by thymic peptide: Pidotimod(PTD). Hum Vaccin Immunother 8: 1250-1258, 2012.

16. Hu S, Fu X, Fu A, Wei D, Ji J and Li W: The regulatory peptide pidotimod facilitates M2 macrophage polarization and its function. Amino Acids 46: 1177-1185, 2014.

17. Shen HH, Ochkur SI, McGarry MP, Crosby JR, Hines EM, Borchers MT, Wang H, Biechelle TL, O'Neill KR, Ansay TL, et al: A causative relationship exists between eosinophils and the development of allergic pulmonary pathologies in the mouse. J Immunol 170: 3296-3305, 2003.

18. Tong J, Bandulwala HS, Clay BS, Anders RA, Shilling RA, Balachandran DD, Chen B, Weinstock JV, Solway J, Hamann KJ and Sperling AI: Fas-positive $\mathrm{T}$ cells regulate the resolution of airway inflammation in a murine model of asthma. J Exp Med 203: 1173-1184, 2006.

19. Bandukwala HS, Clay BS, Tong J, Mody PD, Cannon JL, Shilling RA, Verbeek JS, Weinstock JV, Solway J and Sperling AI: Signaling through Fc gamma RIII is required for optimal Thelper type (Th)2 responses and Th2-mediated airway inflammation. J Exp Med 204: 1875-1889, 2007.

20. Barnes PJ: Th2 cytokines and asthma: An introduction. Respir Res 2: 64-65, 2001.

21. Wang J, Fei LI, Yang M, Wu J, Zhao J, Gong W, Liu W, Bi W and Dong L: FIZZ1 promotes airway remodeling through the PI3K/Akt signaling pathway in asthma. Exp Ther Med 7: 1265-1270, 2014

22. Vonk JM, Postma DS, Maarsingh H, Bruinenberg M, Koppelman GH and Meurs H: Arginase 1 and arginase 2 variations associate with asthma, asthma severity and beta 2 agonist and steroid response. Pharmacogenet Genomics 20: 179-186, 2010.

23. Hankin CS and Cox L: Allergy immunotherapy: What is the evidence for cost saving? Curr Opin Allergy Clin Immunol 14: 363-370, 2014.

24. Li XM: Treatment of Asthma and Food Allergy With Herbal Interventions From Traditional Chinese Medicine. Mt Sinai J Med 78: 697-716, 2011.

25. Nials AT and Uddin S: Mouse models of allergic asthma: Acute and chronic allergen challenge. Dis Models Mech 1: 213-220, 2008.

26. Gries DM, Moffitt DR, Pulos E and Carter ER: A single dose of intramuscularly administered dexamethasone acetate is as effective as oral prednisone to treat asthma exacerbations in young children. J Pediatr 136: 298-303, 2000.

27. Zhao J, Yeong LH and Wong WS: Dexamethasone alters bronchoalveolar lavage fluid proteome in a mouse asthma model. Int Arch Allergy Immunol 142: 219-229, 2006.

28. Schleimer RP: An overview of glucocorticoid anti-inflammatory actions. Eur J Clin Pharmacol 45 (Suppl 1): S3-S7, 1993.

29. Gourgiotis D, Papadopoulos NG, Bossios A, Zamanis P and Saxoni-Papageorgiou P: Immune modulator pidotimod decreases the in vitro expression of CD30 in peripheral blood mononuclear cells of atopic asthmatic and normal children. J Asthma 41: 285-287, 2004.

30. Cohn L, Homer RJ, MacLeod H, Mohrs M, Brombacher F and Bottomly K: Th2-induced airway mucus production is dependent on IL-4Ralpha, but not on eosinophils. J Immunol 162: 6178-6183, 1999. 
31. Taube C, Duez C, Cui ZH, Takeda K, Rha YH, Park JW, Balhorn A, Donaldson DD, Dakhama A and Gelfand EW: The role of IL-13 in established allergic airway disease. J Immunol 169: 6482-6489, 2002.

32. Holcomb IN, Kabakoff RC, Chan B, Baker TW, Gurney A, Henzel W, Nelson C, Lowman HB, Wright BD, Skelton NJ, et al: FIZZ1, a novel cysteine-rich secreted protein associated with pulmonary inflammation, defines a new gene family. EMBO J 19: 4046-4055, 2000.

33. Dong L, Wang SJ, Camoretti-Mercado B, Li HJ, Chen M and Bi WX: FIZZ1 plays a crucial role in early stage airway remodeling of OVA-induced asthma. J Asthma 45: 648-653, 2008.

34. Baldwin L and Roche WR: Does remodelling of the airway wall precede asthma? Paediatr Respir Rev 3: 315-320, 2002.

35. Teng X, Li D, Champion HC and Johns RA: FIZZ1/RELMalpha, a novel hypoxia-induced mitogenic factor in lung with vasoconstrictive and angiogenic properties. Circ Res 92: 1065-1067, 2003.

36. Zhang L, Wang M, Kang X, Boontheung P, Li N, Nel AE and Loo JA: Oxidative stress and asthma: Proteome analysis of chitinase-like proteins and FIZZ1 in lung tissue and bronchoalveolar lavage fluid. J Proteome Res 8: 1631-1638, 2009.

37. Knippenberg S, Brumshagen C, Aschenbrenner F, Welte T and Maus UA: Arginase 1 activity worsens lung-protective immunity against Streptococcus pneumoniae infection. Eur J Immunol 45 $1716-1726,2015$
38. Maarsingh $\mathrm{H}, \mathrm{Zaagsma} \mathrm{J}$ and Meurs $\mathrm{H}$ : Arginase: A key enzyme in the pathophysiology of allergic asthma opening novel therapeutic perspectives. Br J Pharmacol 158: 652-664, 2009.

39. Maarsingh $\mathrm{H}$, Pera $\mathrm{T}$ and Meurs $\mathrm{H}$ : Arginase and pulmonary diseases. Naunyn Schmiedebergs Arch Pharmacol 378: 171-184, 2008.

40. Morris CR, Poljakovic M, Lavrisha L, Machado L, Kuypers FA and Morris SM Jr: Decreased arginine bioavailability and increased serum arginase activity in asthma. Am J Respir Crit Care Med 170: 148-153, 2004.

41. Maarsingh H, Dekkers BG, Zuidhof AB, Bos IS, Menzen MH, Klein T, Flik G, Zaagsma $J$ and Meurs H: Increased arginase activity contributes to airway remodelling in chronic allergic asthma. Eur Respir J 38: 318-328, 2011.

42. Yang M, Rangasamy D, Matthaei KI, Frew AJ,Zimmmermann N, Mahalingam S, Webb DC, Tremethick DJ, Thompson PJ, Hogan SP, et al: Inhibition of arginase I activity by RNA interference attenuates IL-13-induced airways hyperresponsiveness. J Immunol 177: 5595-5603, 2006.

43. North ML, Khanna N, Marsden PA, Grasemann H and Scott JA: Functionally important role for arginase 1 in the airway hyperresponsiveness of asthma. Am J Physiol Lung Cell Mol Physiol 296: L911-L920, 2009. 